\title{
Evaluation of 3D Body Shape Predictions Based on Features
}

\author{
Femke DANCKAERS*, Toon HUYSMANS, Daniël LACKO, Jan SIJBERS \\ iMinds Vision Lab, Dept. of Physics, University of Antwerp, Belgium
}

DOI: $10.15221 / 15.258$ http://dx.doi.org/10.15221/15.258

\begin{abstract}
The human body comes in many sizes and shapes. For design purposes, it is useful to be able to quickly simulate a virtual mannequin of a customer. A statistical shape model can be used for this purpose, because it describes the main variations of body shape inside the model's population. From this model, the specific features of each person in the population are known. Therefore, a mapping between the shape model parameters and specific features can be calculated, which allows adjusting the body shape, in an intuitive way. In this work, we have investigated how accurate a body shape can be predicted based on a set of features and which features are most suitable for this purpose. Height, weight, and hip circumference appeared to be the most suitable features to accurately predict the body shape.
\end{abstract}

Keywords: shape prediction, body features

\section{Introduction}

When designing a near-body product, it is useful to be able to quickly simulate a virtual mannequin of a customer [1]. This mannequin must closely resemble the actual body shape of the customer. Such models (Digital Human Modeling Tools - DHM) are already available [2, 3, 4, 5], but their applicability is still limited. Indeed, the current models are a simplified representation of the body and the 3D variation in shape of the population is usually not included. Moreover, the changes in shape are often univariate (body stature). As a result, they are not suitable for the design of products while focusing on comfort.

Detailed, realistic models are not available yet, but would have an added value for the design process. These models should be accurate, easy to adjust and require a minimum number of parameters $[6,7$, 8].

During the design process, the products could be virtually validated with a broad range of body shapes included in the body model. A statistical shape model [9] of the population is an elegant approach to obtain detailed mannequins that are adjustable in body shape [10,11].

In this work, we evaluate a data-driven technique to extract specific feature parameters from a statistical shape model [12]. We have investigated how accurate a body scan can be predicted based on measured features and which features are most suitable for this purpose.

\section{Materials and Methods}

\subsection{Building a Shape Model}

From the CAESAR database, a sample of the Dutch population is selected. From this subset, a statistical shape model is built to obtain a compact description of the shape variances inside the population. To build such a model, the surfaces must be in correspondence. To correspond the surfaces, we used an elastic surface registration algorithm [13].

\subsubsection{Surface Registration}

In the surface registration part, a reference surface is registered to a target surface, such that the geometric distance between those surfaces becomes minimal while maintaining correspondences. The target surface was a smooth surface with distinct features. The reference surface has been uniformly resampled and made watertight.

First, an initial global rigid registration is obtained. Then, a global rigid registration and an elasticity modulated registration are iteratively repeated. During the iterations, the stiffness gradually decreases, such that the surface will become more elastic through the iterations. The framework is illustrated in Fig. 1 The surface registration framework. 


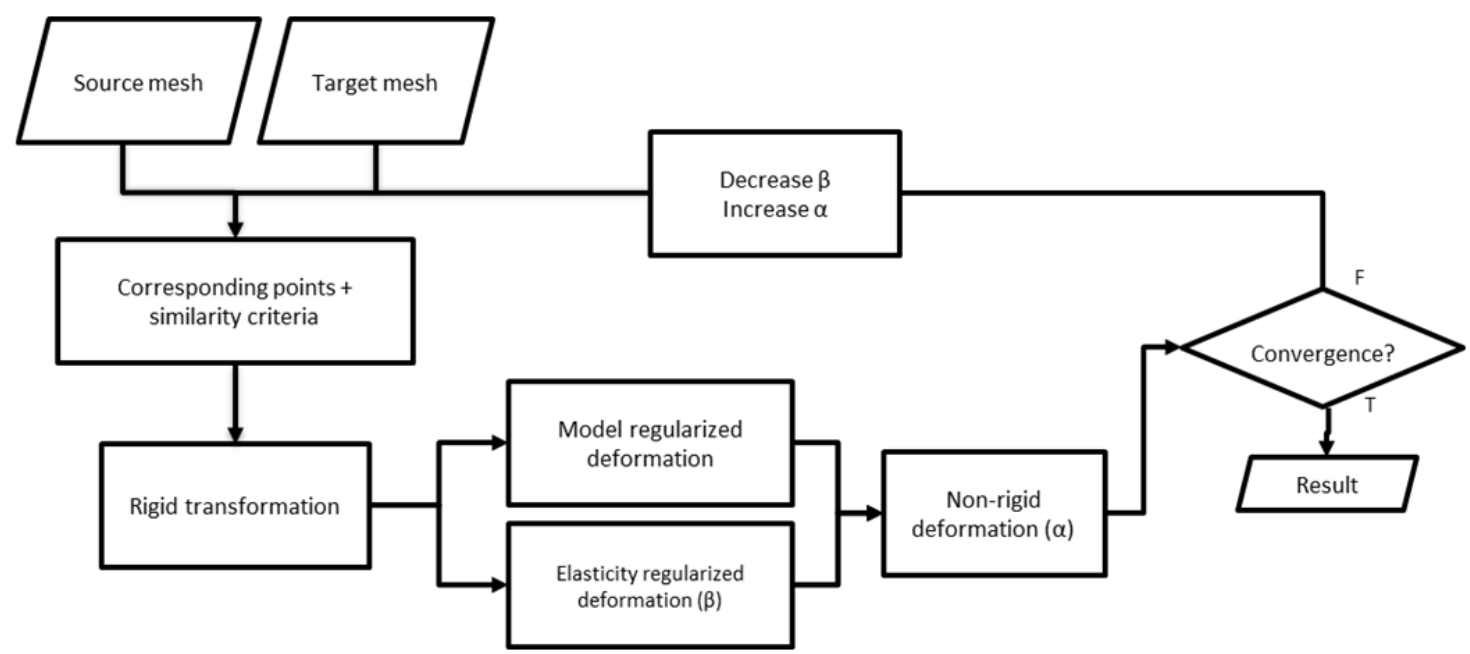

Fig. 1 The surface registration framework

The first step of surface registration is a rigid alignment of the source and target mesh. To that end, in both surfaces corresponding points are identified. This is done by casting a normal ray from each vertex of the reference surface to the target surface. When the normal of an intersection point is in the same direction (within a tolerance) as the normal of the point on the reference surface, those points can be considered corresponding. Another restriction for corresponding points is that the normal may not intersect the surface multiple times before reaching the corresponding point. Those points are used to align the surfaces using a least squares estimation of transformation.

In the elastic part of the registration the vertices are allowed to translate separately, while motion is restricted by a stiffness parameter $\beta$ that regulates the strength of the connection with the neighboring vertices and which decreases during the iterations. In this way, the movement of neighboring vertices is constrained, resulting in similar movements for nearby vertices, as displayed in Fig. 2Fig. 2. By applying weights to each vertex the importance of this vertex can be set. If no corresponding point for a vertex of the target mesh can be found, its weight is set to zero. In that case, this vertex simply translates along with its neighboring vertices.

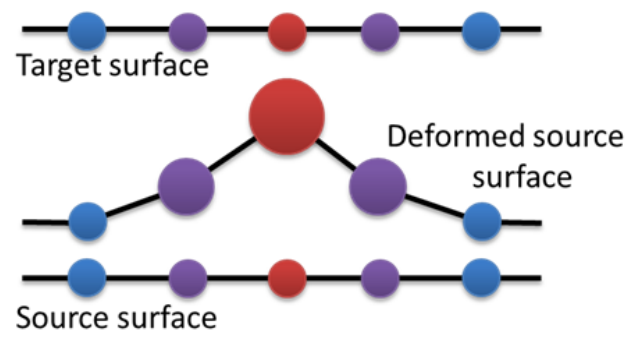

Fig. 2 Stiffness

Finally, a non-rigid deformation is applied for each vertex, regulated by a parameter $\alpha$, which controls the ratio between model regularized deformation and elasticity regularized deformation. Because there is no prior knowledge available from a statistical shape model, only elasticity regularized deformation is applied.

\subsubsection{Building a Statistical Shape Model from Corresponded Surfaces}

A statistical shape model is built from corresponded body scan surfaces using principal component analysis (PCA), describing the average shape and the main variations of the body scan population. To build such a shape model, it is important that the surfaces are superimposed by optimally translating and rotating the surfaces. The optimal transformations are determined by Procrustes analysis. A statistical shape model can be presented with the following formula:

$$
\boldsymbol{Y}=\boldsymbol{C}(\overline{\boldsymbol{X}}+\boldsymbol{\Phi} \boldsymbol{b}) \text {, }
$$

with $\boldsymbol{Y}$ an instance of the model, $\bar{X}$ the average surface, $\boldsymbol{C}$ a rigid transformation and $\boldsymbol{b}$ the shape parameters. Matrix $\boldsymbol{\Phi}$ holds the eigenvectors of the shape model. By adapting the shape parameters, a new, realistic surface can be formed. 


\subsection{Shape prediction}

Each subject of the population has specific shape model parameters and specific features. A link between the shape model parameters and features is sought by multiple multivariate linear regression, so a specific feature is represented by a linear combination of eigenmodes. Such a feature vector can be calculated. As a result, a specific feature, such as the height of a person, can be adapted by multiplying this feature vector with a factor.

A mapping matrix $\boldsymbol{M}$ is built from the principal component weights $\boldsymbol{B}$ and the features of each surface $\boldsymbol{F}$, describing the relationship between them [14].

$$
\boldsymbol{M}=\boldsymbol{B} \boldsymbol{F}^{+}
$$

With this mapping matrix, new principal component weights $\boldsymbol{b}$ can be generated from given features $\boldsymbol{f}=\left[f_{1} f_{2} \ldots f_{n} 1\right]^{T}$.

$$
\boldsymbol{b}=\boldsymbol{M} \boldsymbol{f}
$$

Feature-specific vectors were calculated. By adapting the weights of these vectors, the most plausible body shape is simulated.

We performed two experiments to validate the feature-shape modeling. First, the extracted featurespecific vectors were varied from -3 to +3 standard divisions and visualized on the body as a color map. Second, a body surface was predicted from a subset of its features and compared to the ground truth, to find the most relevant features for body shape prediciton. The comparison was done by calculating the Euclidean distance between the corresponding points of the predicted shape and its ground truth.

\section{Results}

\subsection{Shape Model}

To validate the technique, we used 290 3D scans (145 men and 145 women) from a Dutch population in standing pose from the CAESAR database. The specific features of each person were known. The features used in this paper were gender $(G)$, age $(A)$, height $(H)$, weight $(W)$, waist circumference (WS), chest circumference (C), hip circumference (HP), thigh circumference (T), crotch height (CR), shoulder breadth (S), and breast circumference (B). The measurements are shown in Fig. 3.

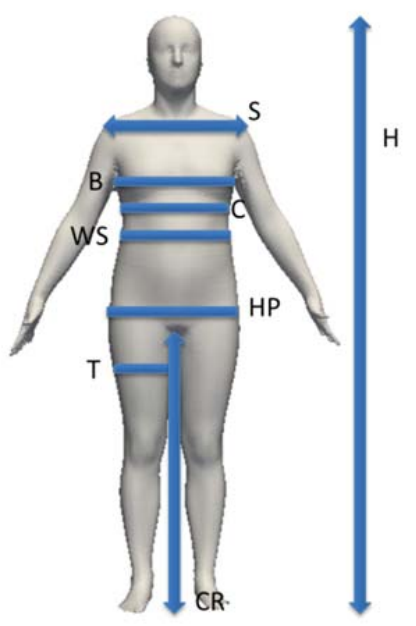

Fig. 3 Features used in this work

The surface registration algorithm was performed for 60 iterations. From the registered surfaces, a statistical shape model was built. The first four shape modes of the CAESAR body model are displayed in Fig. 4. The colors indicate the amount of variation on that location on the surface for the specific eigenmode. The first mode represents mainly height and gender, while the second mode represents mainly weight and gender. It is not possible to link a specific feature with a specific mode. A specific feature consists of a linear combination of multiple eigenmodes. 
mode 1

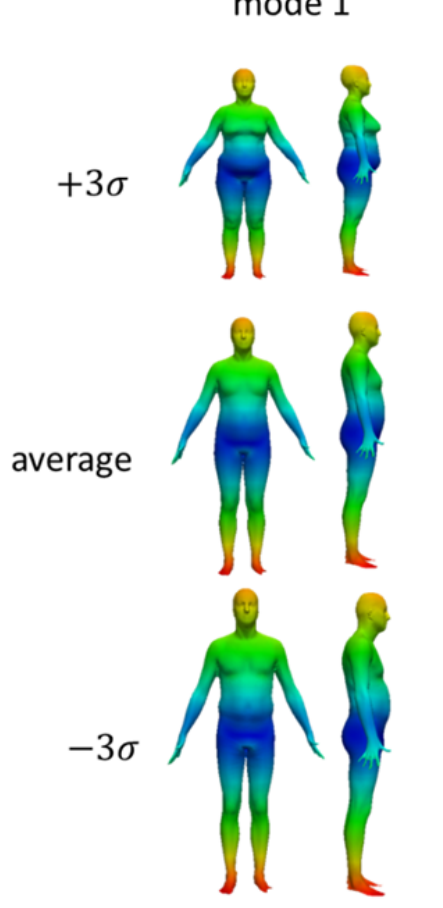

mode 2
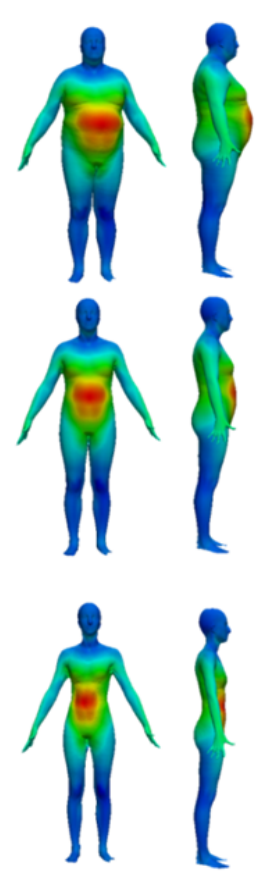

mode 3
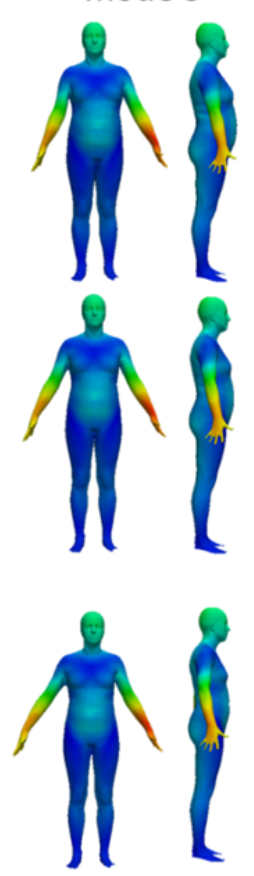

mode 4
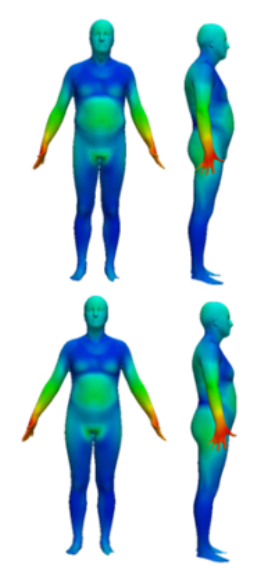

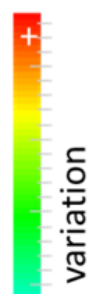

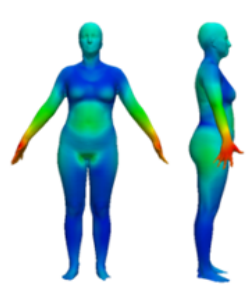

Fig. 4 First four eigenmodes of shape model

The largest variations of the body shape are described in the first modes of the model. This is validated by calculating the compactness of the shape model. The compactness measure describes how the model captures the variation. The compactness test results are visualized in Fig. 5 and shows that the model captures more than $90 \%$ of the shape variation within the first six modes.
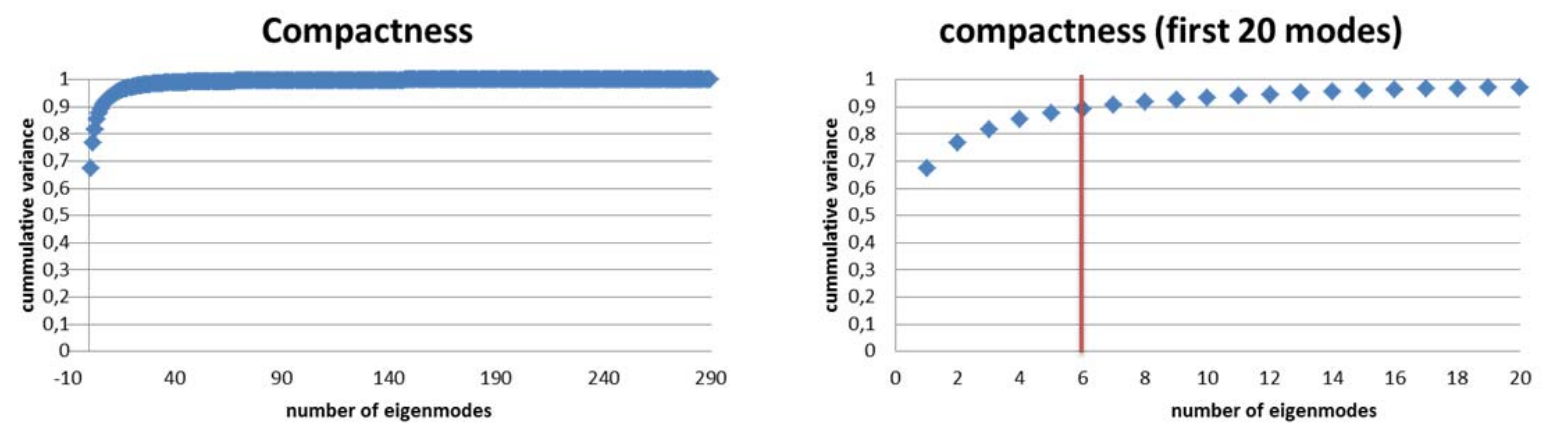

Fig. 5 Compactness

\subsection{Feature Validation}

\subsubsection{Feature vectors}

First, we extracted the feature vectors from the model, using the given features of each sample of the training set. The features of the complete shape model are shown in Fig. 6.

We could observe a difference in gender for extracting the feature vector. In the male population (Fig. 7), a change in weight affects the whole upper body, while in the female population (Fig. 8) weight change is more prominent around the waist. 


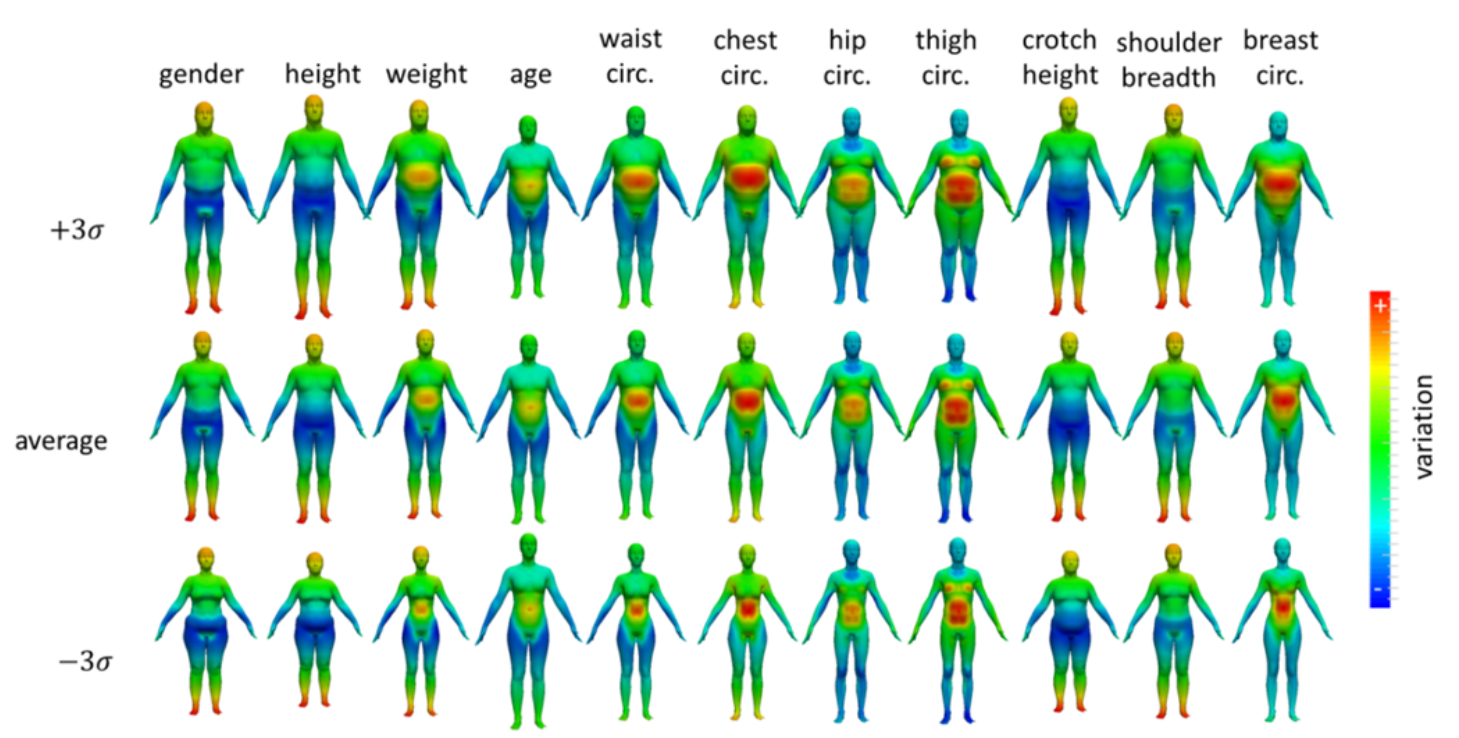

Fig. 6 Feature vectors male + female

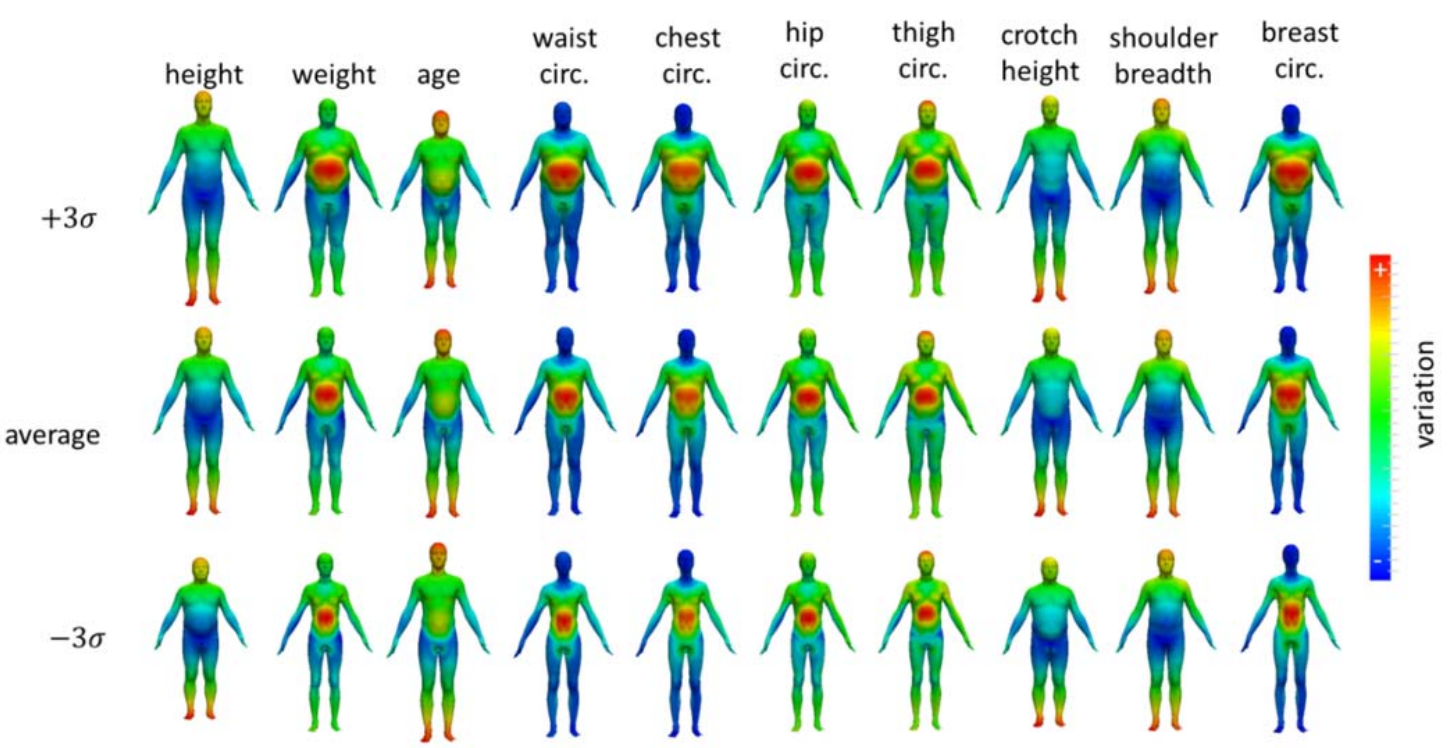

Fig. 7 Feature vectors male
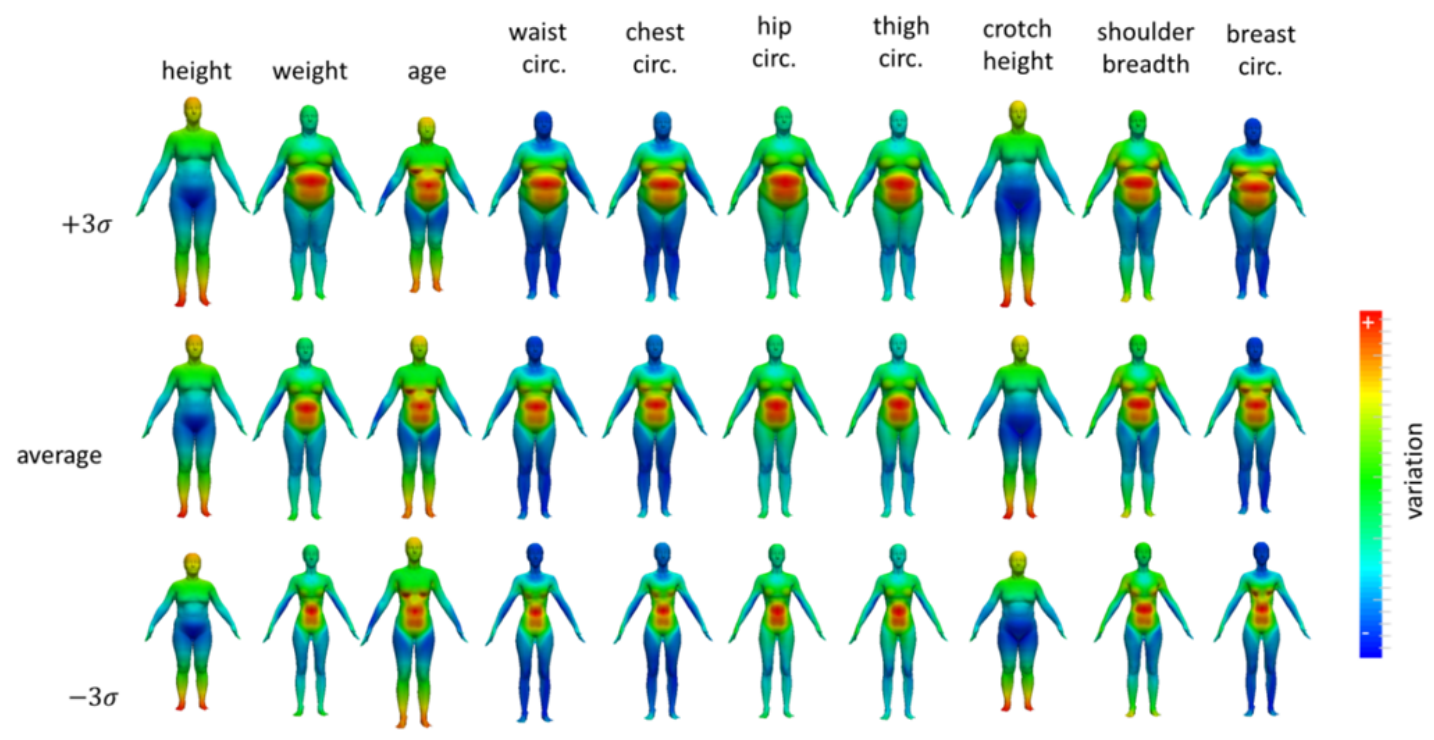

Fig. 8 Feature vectors female 
From these results, we can observe some linear relations between features. Visually, it can be observed that a correlation exist between waist circumference, breast circumference, and chest circumference. There is also a correlation between gender and height. However, no correlation can be observed between breast circumference and height.

In Fig. 9, the relationship between the features is displayed as a pair plot. For each pair of features, the Pearson correlation coefficient is calculated.

\begin{tabular}{|c|c|c|c|c|c|c|c|c|c|c|}
\hline Gender & 0.64 & 0.44 & 0.13 & 0.41 & 0.49 & 0.14 & 0.2 & 0.45 & 0.49 & 0.19 \\
\hline & Height & 0.57 & 0.16 & 0.39 & 0.42 & 0.18 & 0.11 & 0.91 & 0.47 & 0.21 \\
\hline & & Weight & 0.15 & 0.9 & 0.89 & 0.74 & 0.65 & 0.39 & 0.63 & 0.85 \\
\hline & & & Age & 0.32 & 0.27 & 0.064 & 0.051 & 0.22 & 0.041 & 0.28 \\
\hline & & & & $\begin{array}{l}\text { Waist } \\
\text { circ. }\end{array}$ & 0.85 & 0.64 & 0.53 & 0.21 & 0.57 & 0.87 \\
\hline & & & & & $\begin{array}{l}\text { Chest } \\
\text { circ. }\end{array}$ & 0.56 & 0.47 & 0.22 & 0.64 & 0.9 \\
\hline & & & & & & $\begin{array}{l}\text { Hip } \\
\text { circ. }\end{array}$ & 0.89 & 0.1 & 0.33 & 0.67 \\
\hline & & & & & & & $\begin{array}{l}\text { Thigh } \\
\text { circ. }\end{array}$ & 0.053 & 0.26 & 0.57 \\
\hline & & & & & & 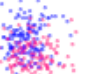 & W & $\begin{array}{l}\text { Crotch } \\
\text { height }\end{array}$ & 0.32 & 0.061 \\
\hline & & & & & & & & & $\begin{array}{l}\text { Shoulder } \\
\text { breadth }\end{array}$ & 0.53 \\
\hline & & & & $x$ & & & & & 4 & $\begin{array}{l}\text { Breast } \\
\text { circ. }\end{array}$ \\
\hline
\end{tabular}

Fig. 9 Pair plot of features. Pink denotes female subjects and blue denotes male subjects. For each pair of features, the Pearson coefficient is displayed.

From the results of the pair plot we can conclude that there is indeed a high correlation between waist circumference, breast circumference, and chest circumference. However, age has little influence on the overall body shape, because there is no significant correlation with any other feature. Height, too, has little influence on the shape since it is only correlated with crotch height.

Furthermore, a difference in gender can be distinguished. For the male population, there is a very high correlation between chest circumference and breast circumference. However, the correlation is less distinct for the female population. Another notable difference is that for the same weight, women have a larger hip circumference and thigh circumference than men, which could also be visually observed from the previous figures. 


\subsubsection{Shape Prediction}

This experiment is performed to find the most useful features to predict a body shape. In Fig. 10, some of the predicted shapes from the test set are displayed. All features were used to predict these surfaces.

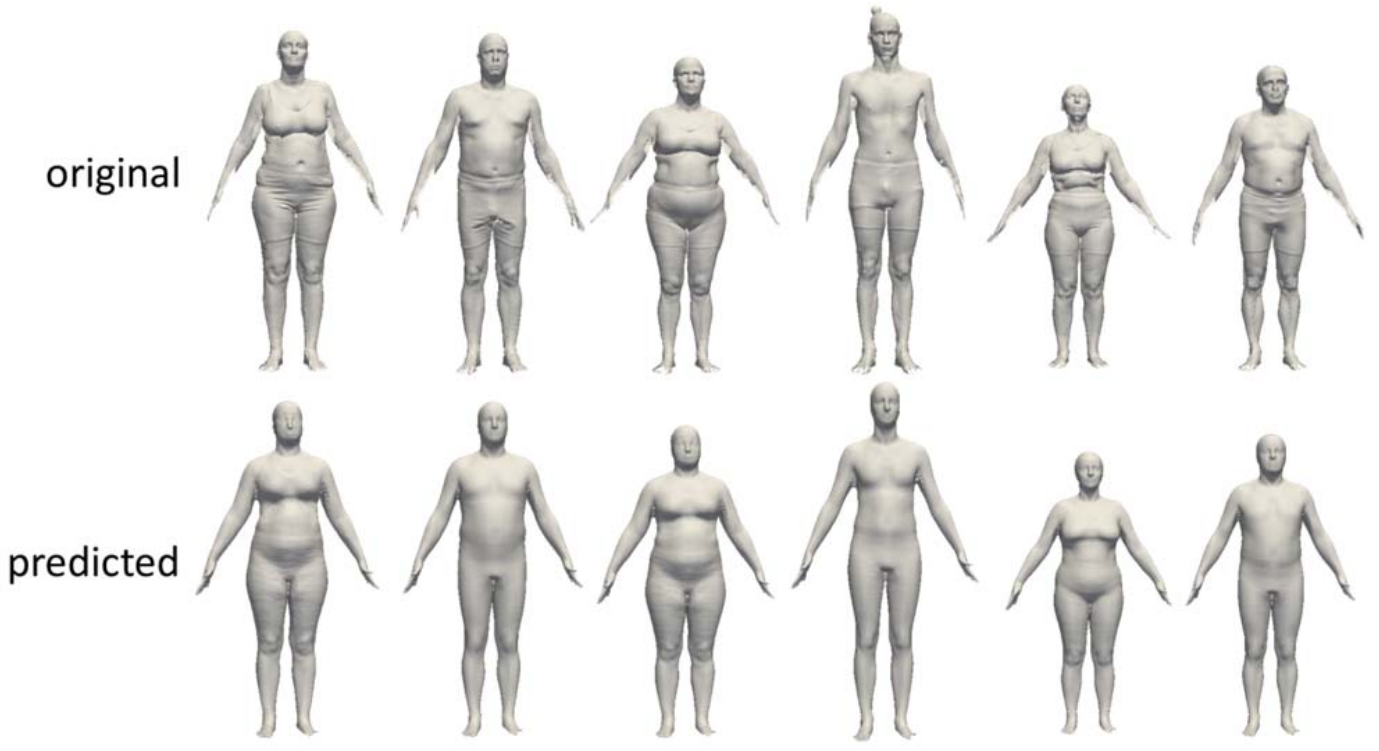

Fig. 10 Examples of predicted shapes

This experiment is performed with 10 subjects ( 5 men and 5 women), not included in the training set. Each surface is predicted from a subset of its features. The predicted surface is compared to the original surface, by calculating the Euclidean distance between both surfaces as a better prediction results in a smaller Euclidean distance. The best performing features were calculated by predicting the surface from all possible combinations of its features. The prediction errors for the optimal combinations are summarized in Table 1.

\begin{tabular}{|c|c|c|c|c|c|c|c|c|c|}
\hline $\mathrm{H}$ & $\mathrm{H}, \mathrm{W}$ & $\begin{array}{c}\mathrm{H}, \mathrm{W}, \\
\mathrm{HP}\end{array}$ & $\begin{array}{c}\text { G,H, } \\
\text { W,HP }\end{array}$ & $\begin{array}{c}\mathrm{G}, \mathrm{H}, \\
\mathrm{W}, \mathrm{HP} \\
\mathrm{S}\end{array}$ & $\begin{array}{l}\mathrm{G}, \mathrm{H}, \\
\mathrm{W}, \mathrm{C}, \\
\mathrm{HP}, \mathrm{S}\end{array}$ & $\begin{array}{c}\mathrm{G}, \mathrm{H}, \mathrm{W}, \\
\mathrm{A}, \mathrm{HP}, \\
\mathrm{CR}, \mathrm{S}\end{array}$ & $\begin{array}{c}\mathrm{G}, \mathrm{H}, \mathrm{W}, \\
\mathrm{A}, \mathrm{HP}, \\
\mathrm{T}, \mathrm{CR}, \mathrm{S}\end{array}$ & $\begin{array}{c}\mathrm{G}, \mathrm{H}, \mathrm{W}, \\
\mathrm{A}, \mathrm{C}, \\
\mathrm{HP}, \mathrm{T} \\
\mathrm{CR}, \mathrm{S}\end{array}$ & $\begin{array}{c}\text { G,H,W, } \\
\text { A,WS, } \\
\text { C,HP, } \\
T, C R, S\end{array}$ \\
\hline $\begin{array}{c}12.16 \\
+3.32\end{array}$ & $\begin{array}{c}10.34 \\
+2.47\end{array}$ & $\begin{array}{c}9.25 \\
\pm 2.18\end{array}$ & $\begin{array}{c}9.10 \\
\pm 2.09\end{array}$ & $\begin{array}{c}9.07 \\
\pm 2.04\end{array}$ & $\begin{array}{c}9.17 \\
\pm 1.96\end{array}$ & $\begin{array}{c}9.09 \\
\pm 2.27\end{array}$ & $\begin{array}{c}9.17 \\
\pm 2.31\end{array}$ & $\begin{array}{r}9.18 \\
\pm 2.17 \\
\end{array}$ & $\begin{array}{c}9.24 \\
\pm 2.25\end{array}$ \\
\hline
\end{tabular}

Table 1. Mean error when predicting a surface using optimal combination of features (in $\mathrm{mm}$ ). $\mathrm{G}=$ gender, $H=$ height, $W=$ weight circumference, $A=$ age, $W S=$ waist, $C=$ chest circumference, $H P=$ hip circumference, $T=$ thigh circumference, $C R=$ crotch height, $S=$ shoulder breadth, $B=$ breast circumference

We can deduct from the results in Table 1 that height, weight, and hip circumference are important features to predict the body shape. Breast circumference and thigh circumference have little influence on the quality of the predictions, or even lead to worse shape predictions due to overfitting. The mean surface errors for above predictions, projected on the average body are visualized in Fig. 11.

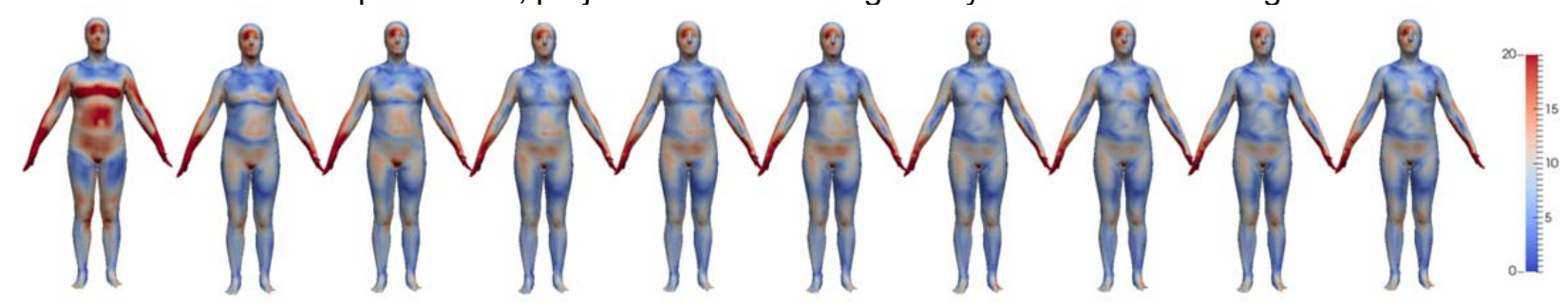

Fig. 11 Mean errors for predicted surfaces of Table 1 in $\mathrm{mm}$

The largest errors appear around the arms, due to differences in pose. Pose normalization is not included in the shape model. 


\section{Conclusion}

In this work, we built a shape model that is adjustable in shape by intuitive parameters.

Strong correlations exist between gender and height, and waist circumference, breast circumference and chest circumference, whereas no correlation was observed between breast circumference and height.

With this algorithm, it is possible to predict the most plausible shape. Height, weight, and hip circumference are the most relevant features to predict the shape of a person. The largest errors appear at the arms, due to differences in pose. In future work, the prediction will be improved by incorporating pose normalization in the shape model.

\section{References}

[1] J. Domingo, M. Ibáñez, A. Simó, G. Ayala en S. Alemany, „Modeling of female human body shapes for apparel design based on cross mean sets," Expert Systems with Applications, vol. 41, nr. 14, pp. 6224-6234, 2014.

[2] P. Blanchonette, „Jack Human Modelling Tool: a Review,” Air Operations Division. Defence Science and Technology Organisation, Tech. Rep. DSTO TR 2364, 2010.

[3] N. Moes, „Digital human models: an overview of development and applications in product and workplace design," in TMCE, Ancona, Italy, 2010.

[4] P. van der Meulen en A. Seidl, „RAMSIS - The Leading Cad Tool for Ergonomic Analysis of Vehicles," Lecture Notes in Computer Science, vol. 4561, pp. 1008-1017, 2007.

[5] B. Park en M. Reed, „Parametric Body Shape Model of Standing Children Aged 3-11 Years.," Ergonomics, 2015.

[6] Y. Otake, C. Carnael, B. Lucas, G. Thawait, J. Carrino, B. Corner, M. Carboni, B. DeCristofano, M. Maffeo, A. Merkle en M. Armand, „Supervised learning of anatomical structures using demographic and anthropometric information," Pattern Recognition Applications and Methods, vol. 318, pp. 225-240, 2014.

[7] X. Ma en A. Luximon, „3D Foot Prediction Method for Low Cost Scanning,” International Journal of Industrial Ergonomics, vol. 44, nr. 6, pp. 866-873, 2014.

[8] S. Wuhrer en C. Shu, „Estimating 3D human shapes from measurements,” Machine Vision and Applications, vol. 24, nr. 6, pp. 1133-1147, 2013.

[9] T. Cootes, C. Taylor, D. Cooper en J. Graham, „Active shape models-their training and application," Computer vision and image understanding, pp. 38-59, 1995.

[10] R. Blanc, C. Seiler, G. Székely, L. Nolte en M. Reyes, „Statistical model based shape prediction from a combination of direct observations and various surrogates: application to orthopaedic research," Medical Image Analysis, vol. 16, nr. 6, pp. 1156-1166, 2012.

[11] D. Lacko, T. Huysmans, T. Parizel, G. De Bruyne, S. Verwulgen, M. Van Hulle en J. Sijbers, „Evaluation of an anthropometric Shape Model of the Human Scalp," Applied Ergonomics, vol. 48, pp. 70-85, 2015.

[12] Z. Liu, J. Li en G. Chen, „Predicting Detailed Body Sizes by Feature Parameters,” International Journal of Clothing Science and Technology, vol. 26, nr. 2, pp. 118-130, 2014.

[13] F. Danckaers, T. Huysmans, D. Lacko, A. Ledda, S. Verwulgen, S. Van Dongen en J. Sijbers, "Correspondence Preserving Elastic Surface Registration with Shape Model Prior," in ICPR, 2014.

[14] B. Allen, B. Curless en Z. Popovic, „The Space of Human Body Shapes: Reconstruction and Parameterization," ACM T, vol. 3, nr. 22, pp. 587-594, 2003. 